\title{
NO'TES ON FOURIER ANALYSIS (XLVI): A CONVERGENCE CRITERION FOR FOURIER SERIES
}

\author{
Gen-ICHIRô SunOUCHI
}

(Received October 30, 1950)

1. Introduction. The object of this paper is to generalize Young's convergence criterion for Fourier series. To simplify the writing, we shall suppose that the Fourier series

$$
\varphi(t) \sim \frac{1}{2} a_{0}+\sum_{n=1}^{\infty} a_{n} \cos n t
$$

in question is that of an even periodic function which is integrable in the Lebesgue sense. Then Pollard [4] generalizes Young's test as follows.

THEOREM. The Fourier series of $\varphi(t)$ converges at the point $t=0$ to the value zero, provided that

$$
\int_{0}^{t} \varphi(u) d u=o(t), \quad \text { as } t \rightarrow 0
$$

and

$$
\int_{0}^{t}|d\{u \varphi(u)\}|=O(t), \quad 0 \leqq t \leqq \eta .
$$

On the other hand Hardy and Littlewood [1] proposed the problem, whether we can replace (1) and (2) by

$$
\int_{0}^{t} \varphi(u) d u=o\left(t / \log \frac{1}{t}\right), \text { as } t \rightarrow 0
$$

and

$$
\int_{0}^{t}\left|d\left\{u^{\Delta} \varphi(u)\right\}\right|=O(t), 0 \leqq t \leqq \eta,
$$

for some $\Delta>1$. Later Randels $[5]$ proved that this is impossible. Concerning this problem we shall prove the following theorem.

ThEOREM. The Fourier series of $\varphi(t)$ converges at the point $t=0$ to the value zero, provided that there is $a \Delta \geqq 1$ such that

$$
\int_{0}^{t} \varphi(u) d u=o\left(t^{\Delta}\right), \quad \text { as } t \rightarrow 0,
$$

and

$$
\int_{0}^{t}\left|d\left\{u^{\Delta} \varphi(u)\right\}\right|=O(t), \quad 0 \leqq t \leqq \eta .
$$

2. Proof of Theorem. It is sufficient to prove that 


$$
\lim _{\omega \rightarrow \infty} \int_{0}^{\pi} \varphi(t) \frac{\sin \omega t}{t} d t=0
$$

Since $\varphi(t)$ is Lebesgue integrable, we have

$$
\lim _{\omega \rightarrow \infty} \int_{\eta}^{\pi} \varphi(t) \frac{\sin \omega t}{t} d t=0
$$

for any fixed $\eta>0$. Let us now put

$$
\alpha=(k / \omega)^{1 / \Delta}
$$

where $k$ is a constant taken sufficiently large and put

$$
\Phi(t)=\int_{0}^{t} \varphi(u) d u=o\left(t^{\Delta}\right), \quad \text { as } t \rightarrow 0 .
$$

Then we have

say, where

$$
\begin{aligned}
\int_{0}^{\alpha} \varphi(t) \frac{\sin \omega t}{t} d t & =\left[\Phi(t) \frac{\sin \omega t}{t}\right]_{0}^{\alpha}-\int_{0}^{\alpha} \Phi(t) \frac{\omega t \cos \omega t-\sin \omega t d t}{t^{2}} \\
& =I_{1}+I_{2},
\end{aligned}
$$

and

$$
\left|I_{1}\right|=o\left(\alpha^{\Delta-1}\right)=o\left\{(k / \omega)^{(\Delta-1) / \Delta}\right\}=o(1), \text { as } \omega \rightarrow \infty
$$

$$
\begin{aligned}
\left|I_{2}\right|=o\left(\omega \int_{0}^{\alpha} t^{\Delta-1}\right) & =o\left(\omega \alpha^{د}\right)=o\left\{\omega(k / \omega)^{\Delta / \Delta}\right\} \\
& =o(1), \text { as } \omega \rightarrow \infty .
\end{aligned}
$$

Hence it is sufficient to prove that

$$
\lim _{h \rightarrow \infty} \lim _{\omega \rightarrow \infty}\left|\int_{a}^{\eta} \varphi(t) \frac{\sin \omega t}{t} d t\right|=0
$$

where $\quad \alpha=(k / \omega)^{1\lrcorner}$.

Let us put $\theta(t)=t^{\Delta} \varphi(t)$ and $\Theta(t)=\int_{0}^{t}|d \theta(u)|$, then $\Theta(t)=O(t)$ and $\theta(t)=O(t)$, since $\theta(0)=0$ is an easy consequence of (5) and (6).

Our concerning integral is therefore

$$
\begin{aligned}
J & =\int_{\alpha}^{\eta} \varphi(t) \frac{\sin \omega t}{t} d t=\int_{\alpha}^{\eta} \theta(t) \frac{\sin \omega t}{t^{\Delta+1}} d t \\
& =-\int_{\alpha}^{\eta} \theta(t) d \Lambda(t),
\end{aligned}
$$

where

$$
\Lambda(t)=\int_{t}^{\eta} \frac{\sin \omega t}{t^{\Delta+1}} d t
$$


From the second mean value theorem, we get

$$
\Lambda(t)=\frac{1}{t^{\Delta+1}} \int_{t}^{\xi} \sin \omega t d t=O\left\{\omega^{-1} t^{-(\Delta+1)}\right\}
$$

Then

$$
\begin{aligned}
-J & =\int_{\alpha}^{\eta} \theta(t) d \Lambda(t)=[\theta(t) \Lambda(t)]_{\alpha}^{\eta}+\int_{\alpha}^{\eta} \Lambda(t) d \theta(t) \\
& =J_{1}+J_{2},
\end{aligned}
$$

say. We have now

$$
\begin{aligned}
J_{1} & =O\left(\omega^{-1} \alpha^{-\Delta}\right)=O\left\{\omega^{-1}(k / \omega)^{-\Delta / \Delta}\right\} \\
& =O\left(k^{-1}\right)=o(1), \quad \text { as } k \rightarrow \infty,
\end{aligned}
$$

and

say, where

$$
\begin{aligned}
J_{2} & =\int_{\infty}^{\eta}|\Lambda(t)||d \theta(t)|=\omega^{-1} \int_{\alpha}^{\eta} O\left\{t^{-(\Delta+1)}\right\} \mid d \theta(t) \\
& =O\left\{\omega^{-1}\left[t^{-(\Delta+1)} \Theta(t)\right]_{\alpha}^{\eta}\right\}+O\left\{\omega^{-1} \int_{\alpha}^{\eta} \Theta(t) t^{-(\Delta+2)} d t\right\}^{\prime} \\
& =K_{1}+K_{2}
\end{aligned}
$$
and

$$
\left.K_{1}=O\left(\omega^{-1}\right)+O\left(\omega^{-1} \alpha^{-\Delta}\right)=o(1)+O\left\{\omega^{-1}(k / \omega)^{-1}\right\}=O^{\prime} k^{-1}\right)=o^{\prime}(1), \quad \text { as } k \rightarrow \infty
$$

$$
K_{2}=O\left\{\omega^{-1} \int_{\alpha}^{\eta} t^{-(\Delta+1)} d t\right\}=O\left(\omega^{-1}\left[t^{-\Delta}\right]_{\alpha}^{\eta}\right)=o(1) .
$$

Thus we get the theorem.

REMARK 1. The condition (5) does not imply the convergence of the Fourier series of $\boldsymbol{\varphi}(\boldsymbol{t})$. See Hsiang [2] or Izumi and Sunouchi [3].

REMARK 2. If (5) and (6) is valid for $0 \leqq t \leqq \eta$, then the analogous estimation gives

$$
a_{n}=\int_{0}^{\pi} \varphi(t) \cos n t d t=O\left(n^{-1 / \Delta}\right)
$$

provided that $\Delta>1$. Hence our test is closely connected with the test of Wang[6].

\section{LITERATURE}

1. G. H. HARDY AND J.E. litTTLEwood, Some new convergence criteria for Fourier series, Annali di Pisa, 3 (1934), 43-62.

2. F. C. Hsiang, The summability $(C, 1-\varepsilon)$ of Fourier series, Duke Math. Journ., 13 (1946), 43-50.

3. S. Izumi and G. Sunotchi, Theorems concerning Cesàro summability, Tôboku Math. Journ. , 1 (2) (1950), 313-326. 
4. S. Pollard, Criteria for convergence of a Fourier series, Journ. London Math. Soc., 2 (1今27), 255-262.

5. W. C. Randels, Three examples in the theory of Fourier series, Annals of Math. , 36 (1935), 835-858.

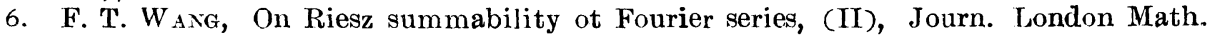
Soc., 17 (1942), 98-107.

Mathematical Institute, Tôhokd University, Sendai. 